\title{
Trends in Racial Disparities in Pancreatic Cancer Surgery
}

\author{
Anand Shah • K. S. Clifford Chao • Truls Østbye • \\ Anthony W. Castleberry • Ricardo Pietrobon • Beat Gloor • \\ Bryan M. Clary • Rebekah R. White • Mathias Worni
}

Received: 14 February 2013 / Accepted: 29 July 2013 /Published online: 4 September 2013

(C) 2013 The Society for Surgery of the Alimentary Tract

\begin{abstract}
Objectives We tested three hypotheses: (1) blacks with pancreatic cancer are recommended surgical resection less often than whites; (2) when recommended surgical resection, blacks refuse surgery more often than whites; and lastly, (3) racial differences in refusal of surgical resection have decreased over time.

Methods A retrospective cohort study was conducted on patients with potentially resectable, nonmetastatic pancreatic adenocarcinoma of the Surveillance, Epidemiology, and End Results registry from 1988 to 2009. Univariate and multivariable logistic regression analyses were performed to assess whether differences in the proportion of whites versus blacks refusing surgery among patients recommended for resection changed over time.

Results A total of 35,944 patients were included; most were white (87.6 \%). After adjusting for covariates including tumor stage, pancreatic cancer resection was less often recommended to and performed in blacks compared with whites (adjusted odds ratio (aOR) $0.88,95 \%$ confidence interval (CI) $0.82-0.95$; aOR $0.83,95 \%$ CI $0.76-0.91$, respectively). Blacks also underwent surgical resection less often when surgery was recommended (aOR $0.73,95 \%$ CI $0.64-0.85$ ). Racial disparities in surgery recommendation and its performance did not decrease from 1988 to 2009. In multivariable adjusted analyses, blacks refused surgery more often when it was recommended (aOR in 1988 4.75, 95 \% CI 2.51-9.01); this disparity decreased over time (aOR 0.93 per year, $95 \%$ CI $0.89-0.97$ ). Conclusions Although racial disparities in pancreatic cancer surgery refusal have diminished over the past two decades, significant disparities in the recommendation and performance of surgery persist. It is likely that both provider- and patientlevel factors have a substantial impact on surgery recommendation and its acceptance. The identification of such factors is critical to design a framework for eliminating disparities in cancer-directed surgery for pancreatic cancer.
\end{abstract}

Keywords Disparity $\cdot$ Race $\cdot$ Ethnicity $\cdot$ Pancreatic adenocarcinoma $\cdot$ Pancreatic cancer $\cdot$ Pancreatic resection

A. Shah · K. S. C. Chao

Department of Radiation Oncology, Columbia University Medical Center, New York, NY, USA

T. Østbye

Department of Community and Family Medicine, Duke University Medical Center, Durham, NC, USA

A. W. Castleberry $\cdot$ R. Pietrobon • B. M. Clary · R. R. White $\cdot$

M. Worni

Department of Surgery, Duke University Medical Center, Durham, NC, USA

B. Gloor $\cdot$ M. Worni $(\bowtie)$

Department of Visceral Surgery and Medicine, Inselspital, University of Bern, Bern CH-3010, Switzerland

e-mail: mathias.worni@duke.edu

\section{Introduction}

Pancreatic cancer is a common cause of cancer mortality in the USA, with more than 40,000 estimated new cases in 2012. ${ }^{1}$ Blacks have a higher incidence of disease compared with whites $^{2}$ and experience poorer median survival. ${ }^{3}$ The reasons for this differential survival are likely multifactorial, including variations in treatment pattern based on socioeconomic status and race. Employment, education, and poverty level are common measures of socioeconomic status and may portend differential access to pancreatic cancer treatment. ${ }^{4}$

Racial differences have been observed in pancreatic cancer treatment patterns. A recent 10-year analysis demonstrated that black and white patients with pancreatic cancer were recommended to undergo pancreatectomy at similar rates, ${ }^{5}$ though black patients refuse recommended surgical intervention more often than whites. ${ }^{5 \cdot 6}$ Nevertheless, it is unknown whether such racial disparities in pancreatic cancer treatment have changed over time. 
Using the Surveillance, Epidemiology, and End Results (SEER) registry from 1988 to 2009, we explored racial disparities in surgical resection for pancreatic cancer. Specifically, we tested three hypotheses: (1) blacks with pancreatic cancer are recommended surgical resection less often than whites; (2) when recommended surgical resection, blacks refuse surgery more often than whites; and lastly, (3) racial differences in refusal of surgical resection have decreased over time.

\section{Methods}

An institutional review board approved this retrospective cohort study of the SEER cancer registry data from 1988 to 2009. ${ }^{7}$ Briefly, SEER is the largest population-based cancer registry in the USA; it encompasses approximately $28 \%$ of the US population. SEER*Stat 7.0.5 (SEER, Bethesda, MD, USA) was used to extract patient information. All patients aged $\geq 18$ years with a malignant tumor of the pancreas were included. We then excluded patients with nonadenocarcinoma tumors which were identified using SEER ICD-O-3 histology codes: 8010 to $8012,8015,8020$ to $8022,8140 / 8141,8143,8147$, $8210 / 8211,8230 / 8231,8260$ to $8263,8440,8450,8452 / 8453$, $8470 / 8471,8480 / 8481,8490,8503 / 8504,8507 / 8508,8510$, $8514,8521,8560,8562$, and 8570 to $8576 .{ }^{8}$ Patients with race other than white or black, including those with unknown race, were excluded from the analyses as were patients with pancreatic cancer noted only on the autopsy/death certificate. Patients with distant metastatic disease were also excluded as they are not candidates for potentially curative tumor resection. All other patients were considered to be potentially eligible for surgery. Resection was defined using SEER pancreas codes for cancer-directed surgery: 10 (local or partial surgical excision of pancreas), 20 (total pancreatectomy with/without splenectomy), 30 (subtotal gastrectomy, duodenectomy with complete or partial pancreatectomy with/without splenectomy [Whipple's operation]), 40 (radical regional [partial] pancreatectomy with lymph node dissection and adjacent soft tissue resection), 50 (pancreatectomy, NOS), and 90 (surgery, NOS).

The following patient characteristics were evaluated: age (continuous), gender, race (white, black), marital status (married, unmarried, unknown), year of diagnosis (grouped as 1988-1997, 1998-2003, 2004-2009 to achieve comparable group size), primary tumor location (head of pancreas, other), tumor T-stage (grouped: T0/1/2, T3, T4, TX), tumor N-stage (N0, N1, NX), presence of multiple tumors (yes, no), tumor resection performed (yes, no, unknown), reason why no cancer resection was performed (refused, other), external beam radiation therapy (EBRT) performed (yes, refused, none/other, unknown), and cause of death (alive at last follow-up, pancreas, other), as well as the following ecological variables (based on census tract information): rural or urban location (rural, urban population $\leq 250,000$, urban population $>250,000$, unknown), less than high school education (percentage), and percentage of families below poverty. Tumor T-stage is available in SEER as a distinct variable starting in 2002. Before then, tumor T-stage is not directly available in SEER and was therefore manually recoded using available SEER variables ("extent of disease"). Because it was not possible to distinguish between $\mathrm{T} 1$ and $\mathrm{T} 2$ tumors, they were grouped together with $\mathrm{T} 0$ as one group. In patients where neoadjuvant therapy or no tumor resection was performed, tumor stage is based on clinical information; for resected cancers, pathological tumor stage is noted.

The primary outcome was the difference in the proportion of whites versus blacks refusing surgery among patients recommended for resection from 1988 to 2009. To assess this, we identified all patients for whom surgery was recommended as well as those who ultimately underwent surgery. We then identified patients for whom surgery was recommended and performed. Lastly, we identified patients who refused surgery when it was recommended. In SEER, this is defined as "patient or patient's guardian refused" surgery. ${ }^{2}$ This distinction is possible using SEER as it provides detailed information regarding why no surgery was performed even though it was recommended.

\section{Statistical Analysis}

Demographic and tumor-related characteristics were compared between whites and blacks using Pearson's Chi-square test for unadjusted estimates among categorical variables (counts, percentage) and two-sample unpaired $t$ tests for continuous variables (mean, standard deviation). Univariate and multivariable logistic regression analyses were performed in order to identify predictors of the following: (1) recommendation for surgery among patients potentially eligible for surgery, (2) performance of surgery among patients potentially eligible for surgery, (3) surgical resection among patients for whom surgery was recommended, and (4) refusal of surgery among patients for whom surgery was recommended. The following variables were considered as potential confounders for the four aforementioned outcomes: race, year of diagnosis, age, gender, marital status, tumor localization, T-stage, N-stage, presence of multiple tumors, radiotherapy, rural/urban location, high school education, and families below poverty; the last three variables are based on census tract information. These variables were selected a priori and included in the multivariable adjusted logistic regression analyses. To examine whether differences in surgery refusal changed over time, an interaction term between race (white, black) and year (continuous) was added to the logistic regression model. The interaction term was only kept in the final model if it was statistically significant. We performed sensitivity analysis for the multivariable analysis, excluding patients with T4 disease and of unknown tumor stage, to account 
for the fact that resectability in locally advanced pancreatic cancer is still of great debate.

Results are presented as odds ratios (OR) with $95 \%$ confidence interval (CI). All statistical analyses were performed using STATA/SE version 11.2 (Stata Corporation, College Station, TX, USA).

\section{Results}

A total of 35,944 patients with nonmetastatic pancreatic cancer were included in this study (Fig. 1). Most subjects were white $(n=31,495,87.6 \%)$. Compared with blacks, white patients were older, more often male and married, and more frequently had a solitary tumor and one that was located in the pancreatic head (Table 1).

\section{Predictors of Surgery Being Recommended}

Pancreatic cancer resection was less often recommended to black (35.0\%) compared with white $(39.4 \%$ ) patients (uOR (unadjusted OR) 0.83 , CI 0.82-0.95, $p<0.001$ ) (Table 2). This finding retained statistical significance after adjustment for several patient and tumor-related characteristics (aOR (adjusted OR) 0.88 , CI $0.82-0.95, p=0.001$ ); this racial disparity did not significantly change over time. In multivariable adjusted analyses, surgery was less often recommended in 2009 compared with 1988, as well as for patients with older age and advanced tumor stage and from areas of higher family poverty. In contrast, surgery was more often recommended to females, married patients, those with lymph node involvement, those with multiple tumors, and patients who refused EBRT.

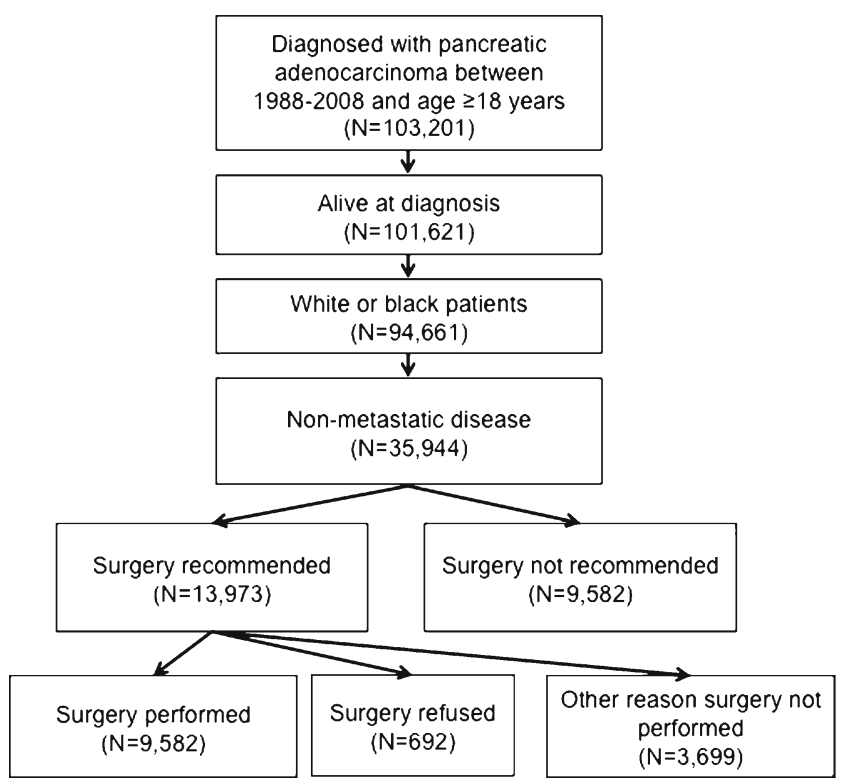

Fig. 1 Study participant flow chart
Predictors of Surgery Being Performed

Among all patients, pancreatic cancer resection was less often performed in black versus white patients (uOR $0.80,95 \% \mathrm{CI}$ $0.74-0.86, p<0.001$ ); this difference did not change over time (Table 3). The significantly lower proportion of pancreatic resections in blacks was still observed after multivariable adjustment (aOR 0.83, CI 0.76-0.91, $p<0.001$ ). However, the odds of surgery performed among all patients with a potentially resectable pancreatic cancer increased from 1988 to 2009 (aOR per year 1.014, CI 1.009-1.020, $p<0.001$ [21.9\% from 1988 to $1997,26.3 \%$ from 1998 to 2003 , $29.5 \%$ from 2004 to 2009]); the interaction between race and year was not significant. Pancreatic resection was more often performed in patients with the following characteristics: female, married, positive lymph nodes, multiple primary tumors, underwent EBRT, and from areas with lower educational attainment. On the other hand, surgery was less often performed in older patients, those with advanced tumor stage, patients who refused EBRT, and those living in areas of higher poverty.

Predictors of Surgical Resection Among Patients Who Were Offered Surgery

Blacks less often underwent surgical resection of pancreatic cancer compared with whites if surgery was recommended (uOR 0.87, CI 0.78-0.97, $p=0.01$ ) (Table 4). While this difference was observed to significantly decrease over time in unadjusted analyses (uOR per year 0.97, CI 0.95-0.99, $p=0.005$ ), no difference was detected over time in covariateadjusted models. The number of resections among patients to whom surgery was offered did increase from 1988 to 2009 (aOR per year 1.07 , CI $1.06-1.08, p<0.001$ ). Surgery was also more frequently performed in patients to whom surgery was offered if they were married, had a T3 tumor or lymph nodepositive disease, had multiple tumors, received EBRT, and resided in areas with lower education levels. Conversely, surgery was less often performed in those with older age, with T4 tumor stage, who refused EBRT, and who resided in an area with higher poverty.

Predictors of Refusal Among Patients Recommended for Surgical Resection

Among patients for whom surgery was recommended, 104 blacks $(6.7 \%)$ refused pancreatic cancer resection compared with 588 whites $(4.7 \%)$ (uOR 1.44, CI 1.16-1.79, $p=0.001$ ) (Table 5). This significant racial difference persisted in adjusted analyses (aOR in 19884.75 , CI 2.51-9.01, $p<0.001$ ) though decreased over time with an aOR of 0.93 per year (CI 0.89 $0.97, p=0.001)$. 
Table 1 Demographic characteristics $(n=35,944)$ overall and by race

\begin{tabular}{|c|c|c|c|c|}
\hline & Overall & $\begin{array}{l}\text { Black } \\
(n=4,449)\end{array}$ & $\begin{array}{l}\text { White } \\
(n=31,495)\end{array}$ & $p$ value \\
\hline Age (mean/SD) & $69.8(12.1)$ & $66.9(12.4)$ & $70.2(12.0)$ & 0.003 \\
\hline \multicolumn{5}{|l|}{ Gender } \\
\hline Male & $17,430(48.5)$ & $1,988(44.7)$ & $15,442(49.0)$ & \multirow[t]{2}{*}{$<0.001$} \\
\hline Female & $18,514(51.5)$ & $2,461(55.3)$ & $16,053(51.0)$ & \\
\hline \multicolumn{5}{|l|}{ Marital status } \\
\hline Married & $19,828(55.2)$ & 1,682 (37.8) & $18,146(57.6)$ & \multirow[t]{3}{*}{$<0.001$} \\
\hline Unmarried & $15,050(41.9)$ & $2,584(58.1)$ & $12,466(39.6)$ & \\
\hline Unknown & $1,066(3.0)$ & $183(4.1)$ & $883(2.8)$ & \\
\hline \multicolumn{5}{|l|}{ Tumor location } \\
\hline Pancreatic head & $25,216(70.2)$ & $3,060(68.8)$ & $22,156(70.3)$ & \multirow[t]{2}{*}{0.03} \\
\hline Other & $10,728(29.8)$ & 1,389 (31.2) & 9,339 (29.7) & \\
\hline \multicolumn{5}{|l|}{ T-stage } \\
\hline $\mathrm{T} 0 / 1 / 2$ & $8,259(23.0)$ & $1,014(22.8)$ & $7,245(23.0)$ & \multirow[t]{4}{*}{$<0.001$} \\
\hline $\mathrm{T} 3$ & $18,750(52.2)$ & $2,183(49.1)$ & 16,567 (52.6) & \\
\hline $\mathrm{T} 4$ & $7,279(20.2)$ & $1,012(22.7)$ & 6,267 (19.9) & \\
\hline $\mathrm{Tx}$ & $1,656(4.6)$ & $240(5.4)$ & $1,416(4.5)$ & \\
\hline \multicolumn{5}{|l|}{$\mathrm{N}$-stage } \\
\hline No & $17,612(49.0)$ & 2,309 (51.9) & 15,303 (48.6) & \multirow[t]{3}{*}{$<0.001$} \\
\hline N1 & $11,146(31.0)$ & $1,252(28.1)$ & 9,894 (31.4) & \\
\hline $\mathrm{Nx}$ & $7,186(20.0)$ & $888(20.0)$ & $6,298(20.0)$ & \\
\hline \multicolumn{5}{|l|}{ Multiple tumors } \\
\hline No & $30,050(83.6)$ & $3,814(85.7)$ & $26,236(83.3)$ & \multirow[t]{2}{*}{$<0.001$} \\
\hline Yes & $5,894(16.4)$ & $635(14.3)$ & $5,259(16.7)$ & \\
\hline \multicolumn{5}{|l|}{ Year of diagnosis } \\
\hline 1988-1997 & $8,545(23.8)$ & $1,079(24.2)$ & $7,466(23.7)$ & \multirow[t]{3}{*}{0.72} \\
\hline 1998-2003 & $11,677(32.5)$ & $1,440(32.4)$ & $10,237(32.5)$ & \\
\hline 2004-2009 & $15,722(43.7)$ & $1,930(43.4)$ & $13,792(43.8)$ & \\
\hline \multicolumn{5}{|l|}{ Tumor resection performed } \\
\hline Yes & $9,582(26.7)$ & $1,025(23.0)$ & $8,557(27.2)$ & \multirow[t]{3}{*}{$<0.001$} \\
\hline No & $26,215(72.9)$ & $3,410(76.7)$ & $22,805(72.4)$ & \\
\hline Unknown & $147(0.4)$ & $14(0.3)$ & $133(0.4)$ & \\
\hline \multicolumn{5}{|l|}{ Radiotherapy (external beam) } \\
\hline Yes & $10,710(29.8)$ & $1,162(26.1)$ & $9,548(30.3)$ & \multirow[t]{4}{*}{$<0.001$} \\
\hline Refused & $571(1.6)$ & $64(1.4)$ & $507(1.6)$ & \\
\hline None/other & $23,960(66.7)$ & 3,141 (70.6) & $20,819(66.1)$ & \\
\hline Unknown & $703(2.0)$ & $82(1.8)$ & $621(2.0)$ & \\
\hline \multicolumn{5}{|l|}{ Rural/urban } \\
\hline Rural & $554(1.5)$ & $28(0.6)$ & $526(1.7)$ & \multirow[t]{4}{*}{$<0.001$} \\
\hline Urban $\leq 250,000$ & $12,871(35.8)$ & $1,198(26.9)$ & $11,673(37.1)$ & \\
\hline Urban $>250,000$ & $22,517(62.6)$ & $3,223(72.4)$ & $19,294(61.3)$ & \\
\hline Unknown & $2(0.0)$ & 0 & $2(0.0)$ & \\
\hline Education below high school (mean, SD) & $20.6(7.4)$ & $23.0(6.8)$ & $20.3(7.4)$ & \multirow[t]{2}{*}{$<0.001$} \\
\hline Unknown & $2(0.0)$ & 0 & $2(0.0)$ & \\
\hline Families below poverty (mean, SD) & $9.2(4.8)$ & $11.7(4.9)$ & $8.9(4.7)$ & \multirow[t]{2}{*}{$<0.001$} \\
\hline Unknown & $2(0.0)$ & 0 & $2(0.0)$ & \\
\hline Cause of death & & & & \\
\hline Alive at last follow-up & $4,256(11.8)$ & $496(11.2)$ & $3,760(11.9)$ & $<0.001$ \\
\hline Pancreatic cancer & $27,768(77.3)$ & $3,334(74.9)$ & $24,434(77.6)$ & \\
\hline Other & $3,920(10.9)$ & $619(13.9)$ & $3,301(10.5)$ & \\
\hline
\end{tabular}


Table 2 Predictors of surgery recommended among 35,944 patients with potentially resectable pancreatic cancer
Reference group: surgery being recommended. In multivariable adjusted analysis, adjustment was performed for gender, marital status, primary tumor localization, T-stage, $\mathrm{N}$-stage, presence of multiple tumors, radiotherapy performance, rural/urban location, high school education, and families below poverty

\begin{tabular}{|c|c|c|c|c|}
\hline & $\begin{array}{l}\text { Surgery } \\
\text { recommended } \\
(n=13,973)\end{array}$ & $\begin{array}{l}\text { Surgery not } \\
\text { recommended } \\
(n=21,971)\end{array}$ & $\begin{array}{l}\text { Multivariable } \\
\text { adjusted } \\
\text { OR }(95 \% \mathrm{CI})\end{array}$ & $p$ value \\
\hline \multicolumn{5}{|l|}{ Race } \\
\hline White & $12,416(39.4)$ & $19,079(60.6)$ & Ref. & \multirow[t]{2}{*}{0.001} \\
\hline Black & $1,557(35.0)$ & $2,892(65.0)$ & $0.88(0.82-0.95)$ & \\
\hline Year of diagnosis & & & $0.976(0.972-0.981)$ & $<0.001$ \\
\hline Interaction race $\times$ year & & & n.s. & n.s. \\
\hline Age (mean, SD) & $67.2(11.8)$ & $71.4(11.9)$ & $0.974(0.972-0.976)$ & $<0.001$ \\
\hline \multicolumn{5}{|l|}{ Gender } \\
\hline Male & $6,900(39.6)$ & $10,530(60.4)$ & Ref. & \multirow[t]{2}{*}{0.003} \\
\hline Female & $7,073(38.2)$ & $11,441(61.8)$ & $1.08(1.02-1.13)$ & \\
\hline \multicolumn{5}{|l|}{ Marital status } \\
\hline Married & $8,340(42.1)$ & $11,488(57.9)$ & $1.19(1.13-1.25)$ & \multirow[t]{3}{*}{$<0.001$} \\
\hline Unmarried & $5,212(34.6)$ & $9,838(65.4)$ & Ref. & \\
\hline Unknown & $421(39.5)$ & $645(60.5)$ & & \\
\hline \multicolumn{5}{|l|}{ Tumor localization } \\
\hline Head & $10,124(40.1)$ & $15,092(59.9)$ & Ref. & \multirow[t]{2}{*}{0.95} \\
\hline Other & $3,849(35.9)$ & $6,879(64.1)$ & $1.00(0.95-1.05)$ & \\
\hline \multicolumn{5}{|l|}{ T-stage } \\
\hline $\mathrm{T} 0 / 1 / 2$ & $3,669(44.4)$ & $4,590(55.6)$ & Ref. & \\
\hline $\mathrm{T} 3$ & $8,535(45.5)$ & $10,215(54.5)$ & $0.90(0.85-0.96)$ & $<0.001$ \\
\hline $\mathrm{T} 4$ & $1,483(20.4)$ & $5,796(79.6)$ & $0.27(0.25-0.29)$ & $<0.001$ \\
\hline TX & $286(17.3)$ & $1,370(82.7)$ & $0.32(0.28-0.37)$ & $<0.001$ \\
\hline \multicolumn{5}{|l|}{$\mathrm{N}$-stage } \\
\hline No & $6,139(34.9)$ & $11,473(65.1)$ & Ref. & \\
\hline $\mathrm{N}+$ & $6,145(55.1)$ & $5.001(44.9)$ & $2.12(2.01-2.24)$ & $<0.001$ \\
\hline $\mathrm{Nx}$ & $1,689(23.5)$ & $5,497(76.5)$ & $0.58(0.54-0.63)$ & $<0.001$ \\
\hline \multicolumn{5}{|l|}{ Multiple tumors } \\
\hline Yes & $2,325(39.4)$ & $3.569(60.6)$ & $1.14(1.07-1.21)$ & \multirow[t]{2}{*}{$<0.001$} \\
\hline No & $11,648(38.8)$ & $18,402(61.2)$ & Ref. & \\
\hline \multicolumn{5}{|l|}{ Radiotherapy (beam) } \\
\hline Yes & $4,754(44.4)$ & $5,956(55.6)$ & $1.00(0.95-1.05)$ & 0.98 \\
\hline Refused & $253(44.3)$ & $318(55.7)$ & $1.46(1.22-1.75)$ & $<0.001$ \\
\hline No & $8,685(36.2)$ & $15,275(63.8)$ & Ref. & \\
\hline Unknown & $281(40.0)$ & $422(60.0)$ & & \\
\hline \multicolumn{5}{|l|}{ Rural/urban } \\
\hline Rural & $196(35.4)$ & $358(64.6)$ & Ref. & \\
\hline Urban $\leq 250,000$ & $5,239(40.7)$ & $7,632(59.3)$ & $1.13(0.92-1.37)$ & 0.24 \\
\hline Urban $>250,000$ & $8,537(37.9)$ & $13,980(62.1)$ & $0.94(0.78-1.15)$ & 0.57 \\
\hline Unknown & $1(50.0)$ & $1(50.0)$ & & \\
\hline Education below high school (mean, SD) & $20.3(7.1)$ & $20.8(7.5)$ & $0.995(0.989-1.000)$ & 0.06 \\
\hline Unknown & $1(50.0)$ & $1(50.0)$ & Ref. & \\
\hline Families below poverty (mean, SD) & $9.0(4.7)$ & $9.4(4.8)$ & $0.986(0.977-0.994)$ & 0.001 \\
\hline Unknown & $1(50.0)$ & $1(50.0)$ & & \\
\hline
\end{tabular}

Sensitivity Analysis Excluding T4 and TX Tumor Stage

All estimates comparing blacks and whites for (a) receipt of a surgery recommendation, (b) surgery being performed, and (c) surgery explicitly offered yet refused remained stable including their significance levels in the main analysis (data not shown).

\section{Discussion}

Several national health-care organizations, such as the National Cancer Institute and Agency for Healthcare Research and Quality, have prioritized the identification and reduction of health-care disparities. ${ }^{9 \cdot 10}$ Understanding the magnitude of 
Table 3 Predictors of surgery performed among all patients with nonmetastatic pancreatic cancer ( $n=35,797$ patients)
Reference group: surgery being performed. In multivariable adjusted analysis, adjustment was performed for gender, marital status, primary tumor localization, $\mathrm{T}$-stage, N-stage, presence of multiple tumors, radiotherapy performance, rural/urban location, high school education, and families below poverty (147 patients with unknown information if surgery was performed $(0.41 \%))$

\begin{tabular}{|c|c|c|c|c|}
\hline & $\begin{array}{l}\text { Surgery } \\
\text { performed } \\
(n=9,582)\end{array}$ & $\begin{array}{l}\text { Surgery not } \\
\text { performed } \\
(n=26,215)\end{array}$ & $\begin{array}{l}\text { Multivariable } \\
\text { adjusted } \\
\text { OR }(95 \% \mathrm{CI})\end{array}$ & $p$ value \\
\hline \multicolumn{5}{|l|}{ Race } \\
\hline White & $8,557(27.3)$ & $22,805(72.7)$ & Ref. & \multirow[t]{2}{*}{$<0.001$} \\
\hline Black & $1,025(23.1)$ & $3,410(76.9)$ & $0.83(0.76-0.91)$ & \\
\hline Year of diagnosis & & & $1.014(1.009-1.020)$ & $<0.001$ \\
\hline Interaction race $\times$ year & & & n.s. & n.s. \\
\hline Age & $65.2(11.3)$ & $71.5(11.9)$ & $0.959(0.957-0.962)$ & $<0.001$ \\
\hline \multicolumn{5}{|l|}{ Gender } \\
\hline Male & $4,870(28.1)$ & $12,486(71.9)$ & Ref. & \multirow[t]{2}{*}{0.005} \\
\hline Female & $4,712(25.6)$ & $13,729(74.4)$ & $1.08(1.02-1.15)$ & \\
\hline \multicolumn{5}{|l|}{ Marital status } \\
\hline Married & $3,193(21.3)$ & $11,798(78.7)$ & $1.40(1.32-1.48)$ & \multirow[t]{3}{*}{$<0.001$} \\
\hline Unmarried & $6,144(31.1)$ & $13,613(68.9)$ & Ref. & \\
\hline Unknown & $245(23.4)$ & 804 (76.6) & & \\
\hline \multicolumn{5}{|l|}{ Tumor location } \\
\hline Head & $7,081(28.2)$ & $18,036(71.8)$ & Ref. & \multirow[t]{2}{*}{1.00} \\
\hline Other & $2,501(23.4)$ & $8,179(76.6)$ & $1.00(0.94-1.06)$ & \\
\hline \multicolumn{5}{|l|}{ T-stage } \\
\hline $\mathrm{T} 0 / 1 / 2$ & $2,455(29.9)$ & $5,756(70.1)$ & Ref. & \\
\hline $\mathrm{T} 3$ & $6,435(34.4)$ & $12,260(65.6)$ & $1.00(0.93-1.06)$ & 0.94 \\
\hline $\mathrm{T} 4$ & $582(8.0)$ & $6,671(92.0)$ & $0.15(0.13-0.17)$ & $<0.001$ \\
\hline $\mathrm{Tx}$ & $110(6.7)$ & $1,528(93.3)$ & $0.18(0.14-0.22)$ & $<0.001$ \\
\hline \multicolumn{5}{|l|}{$\mathrm{N}$-stage } \\
\hline No & $4,156(23.7)$ & $13,373(76.3)$ & Ref. & \\
\hline N1 & $5,216(46.9)$ & $5,898(53.1)$ & $2.55(2.41-2.70)$ & $<0.001$ \\
\hline $\mathrm{Nx}$ & $210(2.9)$ & $6,944(97.1)$ & $0.11(0.09-0.13)$ & $<0.001$ \\
\hline \multicolumn{5}{|l|}{ Multiple tumors } \\
\hline Yes & $1,610(27.4)$ & $4,263(73.4)$ & $1.23(1.15-1.33)$ & \multirow[t]{2}{*}{$<0.001$} \\
\hline No & $7,972(26.6)$ & $21,952(72.6)$ & Ref. & \\
\hline \multicolumn{5}{|l|}{ Radiotherapy (beam) } \\
\hline Yes & $3,780(23.1)$ & $6,918(76.9)$ & $1.18(1.11-1.25)$ & $<0.001$ \\
\hline Refused & $93(16.3)$ & $477(83.7)$ & $0.62(0.48-0.80)$ & \multirow[t]{3}{*}{$<0.001$} \\
\hline No & $5,513(23.1)$ & $18,351(76.9)$ & Ref. & \\
\hline Unknown & $196(29.5)$ & $469(70.5)$ & & \\
\hline \multicolumn{5}{|l|}{ Rural/urban } \\
\hline Rural & $143(26.5)$ & $397(73.5)$ & Ref. & \\
\hline Urban $\leq 250,000$ & $3,457(27.0)$ & $9.347(73.0)$ & $0.92(0.73-1.16)$ & 0.47 \\
\hline Urban $>250,000$ & $5,982(26.6)$ & $16,469(73.4)$ & $0.89(0.71-1.12)$ & 0.32 \\
\hline Unknown & 0 & $2(100.0)$ & & \\
\hline Education below high school (mean, SD) & $20.4(7.3)$ & $20.7(7.4)$ & $1.011(1.005-1.018)$ & 0.001 \\
\hline Unknown & 0 & $2(100.0)$ & & \\
\hline Families below poverty (mean, SD) & $9.3(4.8)$ & $9.0(4.7)$ & $0.969(0.959-0.979)$ & $<0.001$ \\
\hline Unknown & 0 & $2(100.0)$ & & \\
\hline
\end{tabular}

these disparities in pancreatic cancer surgery, especially their trends, can inform efforts to improve health-care access for select populations and aid in future research funding allocation. We therefore examined three specific hypotheses: whether blacks with pancreatic cancer are recommended surgical resection less often than whites, blacks refuse surgery more often than whites when recommended surgery, and finally, that racial differences in refusal of surgical resection have decreased over time. Our analysis of the SEER registry from 1988 to 2009 suggests that blacks with pancreatic cancer are 
Table 4 Predictors of surgical resection among patients who were offered surgery $(n=13,961)$
Reference group: surgery being performed. In multivariable adjusted analysis, adjustment was performed for gender, marital status, primary tumor localization, T-stage, N-stage, presence of multiple tumors, radiotherapy performance, rural/urban location, high school education, and families below poverty $(12$ patients with unknown information if surgery was performed $(0.09 \%))$

\begin{tabular}{|c|c|c|c|c|}
\hline & $\begin{array}{l}\text { Surgery } \\
\text { performed } \\
(n=9,582)\end{array}$ & $\begin{array}{l}\text { Surgery not } \\
\text { performed } \\
(n=4,379)\end{array}$ & $\begin{array}{l}\text { Multivariable } \\
\text { adjusted OR } \\
(95 \% \mathrm{CI})\end{array}$ & $p$ value \\
\hline \multicolumn{5}{|l|}{ Race } \\
\hline White & $8,557(69.0)$ & $3,848(31.0)$ & Ref. & \multirow[t]{2}{*}{$<0.001$} \\
\hline Black & $1,025(65.9)$ & $531(34.1)$ & $0.73(0.64-0.85)$ & \\
\hline Year & & & $1.07(1.06-1.08)$ & $<0.001$ \\
\hline Interaction race $\times$ year & & & n.s. & n.s. \\
\hline Age & $65.2(11.3)$ & $71.8(11.7)$ & $0.955(0.951-0.959)$ & $<0.001$ \\
\hline \multicolumn{5}{|l|}{ Gender } \\
\hline Male & $4,870(70.6)$ & $2,027(29.4)$ & Ref. & \multirow[t]{2}{*}{0.48} \\
\hline Female & $4,712(66.7)$ & $2,352(33.3)$ & $1.03(0.94-1.14)$ & \\
\hline \multicolumn{5}{|l|}{ Marital status } \\
\hline Married & $6,144(73.7)$ & $2,192(26.3)$ & $1.45(1.32-1.60)$ & \multirow[t]{3}{*}{$<0.001$} \\
\hline Unmarried & $3,193(61.3)$ & $2,012(38.7)$ & Ref. & \\
\hline Unknown & $245(58.3)$ & $175(41.7)$ & & \\
\hline \multicolumn{5}{|l|}{ Tumor location } \\
\hline Head & $7,081(70.0)$ & $3,033(30.0)$ & Ref. & \multirow[t]{2}{*}{0.13} \\
\hline Other & $2,501(65.0)$ & $1,346(35.0)$ & $1.08(0.98-1.20)$ & \\
\hline \multicolumn{5}{|l|}{ T-stage } \\
\hline $\mathrm{T} 0 / 1 / 2$ & $2,455(67.0)$ & $1,208(33.0)$ & Ref. & \\
\hline $\mathrm{T} 3$ & $6,435(75.4)$ & $2,096(24.6)$ & $1.20(1.08-1.33)$ & 0.001 \\
\hline $\mathrm{T} 4$ & $582(39.3)$ & $900(60.7)$ & $0.33(0.28-0.39)$ & $<0.001$ \\
\hline $\mathrm{Tx}$ & $110(38.6)$ & $175(61.4)$ & $0.27(0.20-0.39)$ & $<0.001$ \\
\hline \multicolumn{5}{|l|}{$\mathrm{N}$-stage } \\
\hline No & $4,156(67.8)$ & $1,974(32.2)$ & Ref. & \\
\hline N1 & $5,216(84.9)$ & $926(15.1)$ & $2.38(2.16-2.63)$ & $<0.001$ \\
\hline $\mathrm{Nx}$ & $210(12.4)$ & $1,479(87.6)$ & $0.10(0.08-0.11)$ & $<0.001$ \\
\hline \multicolumn{5}{|l|}{ Multiple tumors } \\
\hline Yes & $1,610(69.3)$ & $713(30.7)$ & $1.27(1.12-1.43)$ & \multirow[t]{2}{*}{$<0.001$} \\
\hline No & $7,972(68.5)$ & $3,666(31.5)$ & Ref. & \\
\hline \multicolumn{5}{|l|}{ Radiotherapy (beam) } \\
\hline Yes & $3,780(79.5)$ & $973(20.5)$ & $1.31(1.18-1.45)$ & $<0.001$ \\
\hline Refused & $93(36.8)$ & $160(63.2)$ & $0.29(0.21-0.39)$ & \multirow[t]{3}{*}{$<0.001$} \\
\hline No & $5,513(63.6)$ & $3,162(36.4)$ & Ref. & \\
\hline Unknown & $196(70.0)$ & $84(30.0)$ & & \\
\hline \multicolumn{5}{|l|}{ Rural/urban } \\
\hline Rural & $143(73.0)$ & $53(27.0)$ & Ref. & \\
\hline Urban $\leq 250,000$ & $3,457(66.0)$ & $1,779(34.0)$ & $0.80(0.53-1.21)$ & 0.30 \\
\hline Urban $>250,000$ & $5,982(70.1)$ & 2,546 (29.9) & $0.94(0.62-1.42)$ & 0.77 \\
\hline Unknown & 0 & $1(100.0)$ & & \\
\hline Education below high school (mean, SD) & $20.4(7.3)$ & $20.0(6.8)$ & $1.037(1.026-1.049)$ & $<0.001$ \\
\hline Unknown & 0 & $1(100.0)$ & & \\
\hline $\begin{array}{l}\text { Families below poverty } \\
\quad(\text { mean, SD) }\end{array}$ & $9.0(4.7)$ & $9.0(4.8)$ & $0.960(0.945-0.976)$ & $<0.001$ \\
\hline Unknown & 0 & $1(100.0)$ & & \\
\hline
\end{tabular}

less likely to be recommended for surgery compared with whites. Furthermore, surgery is less likely performed in blacks including among patients to whom surgery is recommended. These disparities did not significantly change over time. We also found that even when surgery was recommended, blacks refused surgical resection more often compared with whites, though this disparity has decreased significantly over the past two decades. 
Table 5 Predictors of refusal among patients recommended for surgical resection $(n=13,961)$
Reference group: surgery not refused. In multivariable adjusted analysis, adjustment was performed for gender, marital status, primary tumor localization, $\mathrm{T}$-stage, $\mathrm{N}$-stage, presence of multiple tumors, radiotherapy performance, rural/urban location, high school education, and families below poverty

\begin{tabular}{|c|c|c|c|c|}
\hline & $\begin{array}{l}\text { Surgery not } \\
\text { refused } \\
(n=13,269)\end{array}$ & $\begin{array}{l}\text { Surgery } \\
\text { refused } \\
(n=692)\end{array}$ & $\begin{array}{l}\text { Multivariable } \\
\text { adjusted OR } \\
(95 \% \mathrm{CI})\end{array}$ & $p$ value \\
\hline \multicolumn{5}{|l|}{ Race } \\
\hline White & $11,817(95.3)$ & $588(4.7)$ & Ref. & \multirow[t]{2}{*}{$<0.001$} \\
\hline Black & $1,452(93.3)$ & $104(6.7)$ & $4.75(2.51-9.01)$ & \\
\hline Year of diagnosis & & & $1.05(1.03-1.08)$ & $<0.001$ \\
\hline Interaction between race $\times$ year & & & $0.93(0.89-0.97)$ & 0.001 \\
\hline Age & $66.8(11.7)$ & $76.3(10.4)$ & $1.07(1.06-1.08)$ & $<0.001$ \\
\hline \multicolumn{5}{|l|}{ Gender } \\
\hline Male & $6,620(96.0)$ & $277(4.0)$ & Ref. & \multirow[t]{2}{*}{0.68} \\
\hline Female & $6,649(94.1)$ & $415(5.9)$ & $0.96(0.80-1.16)$ & \\
\hline \multicolumn{5}{|l|}{ Marital status } \\
\hline Married & $8,055(96.6)$ & $281(3.4)$ & $0.71(0.59-0.86)$ & \multirow[t]{3}{*}{$<0.001$} \\
\hline Unmarried & $4,818(92.6)$ & $387(7.4)$ & Ref. & \\
\hline Unknown & $396(94.3)$ & $24(5.7)$ & & \\
\hline \multicolumn{5}{|l|}{ Tumor location } \\
\hline Head & $9.606(95.0)$ & $508(5.0)$ & Ref. & \multirow[t]{2}{*}{$<0.001$} \\
\hline Other & $3,663(95.2)$ & $184(4.8)$ & $0.68(0.55-0.83)$ & \\
\hline \multicolumn{5}{|l|}{ T-stage } \\
\hline $\mathrm{T} 1 / 2$ & $3,400(92.8)$ & $263(7.2)$ & Ref. & \\
\hline $\mathrm{T} 3$ & $8,237(96.6)$ & $294(3.5)$ & $0.59(0.49-0.72)$ & $<0.001$ \\
\hline $\mathrm{T} 4$ & $1,413(95.3)$ & $69(4.7)$ & $0.64(0.47-0.87)$ & 0.004 \\
\hline $\mathrm{Tx}$ & $219(76.8)$ & $66(23.2)$ & $2.38(1.63-3.47)$ & $<0.001$ \\
\hline \multicolumn{5}{|l|}{$\mathrm{N}$-stage } \\
\hline No & $5,698(93.0)$ & $432(7.0)$ & Ref. & \\
\hline N1 & $6,069(98.8)$ & $73(1.2)$ & $0.21(0.16-0.28)$ & $<0.001$ \\
\hline $\mathrm{Nx}$ & $1,502(88.9)$ & $187(11.1)$ & $1.67(1.33-2.10)$ & $<0.001$ \\
\hline \multicolumn{5}{|l|}{ Radiotherapy (beam) } \\
\hline Yes & $4,669(98.2)$ & $84(1.8)$ & $0.64(0.50-0.82)$ & $<0.001$ \\
\hline Refused & $127(50.2)$ & $126(49.8)$ & $22.7(16.4-31.3)$ & $<0.001$ \\
\hline No & $8,201(94.5)$ & $474(5.5)$ & Ref. & \\
\hline Unknown & $272(97.1)$ & $8(2.9)$ & & \\
\hline \multicolumn{5}{|l|}{ Rural/urban } \\
\hline Rural & $180(91.8)$ & $16(8.2)$ & Ref. & \\
\hline Urban $\leq 250,000$ & $4,983(95.2)$ & $253(4.8)$ & $0.77(0.40-1.50)$ & 0.45 \\
\hline Urban $>250,000$ & $8,105(95.0)$ & $423(5.0)$ & $0.87(0.45-1.68)$ & 0.68 \\
\hline Unknown & $1(100.0)$ & 0 & & \\
\hline Education below high school (mean, SD) & $20.2(7.1)$ & $21.4(7.7)$ & $1.03(1.01-1.05)$ & 0.005 \\
\hline Unknown & $1(100.0)$ & 0 & & \\
\hline Families below poverty (mean, SD) & $8.9(4.7)$ & $9.7(4.8)$ & $0.99(0.96-1.02)$ & 0.47 \\
\hline Unknown & $1(100.0)$ & 0 & & \\
\hline
\end{tabular}

This study adds to and updates the findings of Murphy and colleagues who previously examined racial disparities in pancreatic cancer resection using SEER registry data from 1992 to $2002 .{ }^{5}$ The authors concluded that, although blacks and whites presented with similar disease stage and were similarly recommended for resection, blacks were significantly more likely to refuse recommended surgery. ${ }^{5}$ Our analysis differs from
Murphy in that we examined a longer time interval to evaluate longitudinal trends in recommendation and acceptance of surgery, we focused on the ethical comparison of whites and blacks, we excluded patients with distant metastasis as those patients are still not considered candidates for surgical resection, and we included ecological variables to the multivariable analyses to adjust for such differences between whites and 
blacks. In our study, both univariate and multivariate analyses demonstrated racial disparities in the provision of a recommendation for surgery as well as its acceptance.

Notably, we found that blacks were less likely to be recommended for pancreatic cancer surgery, suggesting that provider-level factors may influence whether surgery is recommended. Bilimoria et al. found that, compared with white patients, blacks encounter longer wait times between the diagnosis of pancreatic cancer and surgery. ${ }^{11}$ Although the reasons for such disparities are unclear, a physician's thinking and behavior may be unconsciously influenced by prejudice depending on patient race. ${ }^{12}$ For example, black patients may be less likely to receive needed cancer information and less likely to receive the name of a cancer specialist. ${ }^{13}$ Lastly, evidence from the orthopedic literature suggests that a provider's decision whether to recommend surgery to black patients may depend on the patient's baseline preference or attitude regarding surgery. ${ }^{14}$

Our results support previous evidence that blacks are more likely to refuse pancreatic cancer-directed surgery. ${ }^{15}$ Specifically, we found that blacks refused recommended pancreatic cancer resection more often than whites, though this disparity decreased significantly over the past two decades. In a recent study of curative surgery for upper gastrointestinal cancers, black race was a predictor of nonsurgical approach. ${ }^{16}$ Interestingly, our results demonstrate that patients who refused surgery were also more likely to refuse EBRT. There are several patient-level reasons that could explain the refusal of recommended surgery. Blacks historically have often expressed distrust for the health-care system. ${ }^{17 \cdot 18}$ Black patients often rate their physician visits as less participatory compared with whites; visits with a provider of the same race are rated as being more participatory. ${ }^{19}$ Lastly, it is unknown whether preexisting factors prior to a patient's evaluation for pancreatic cancer may impact their decision making. In a study of patient's decisions regarding surgery for early stage lung cancer, Cykert and colleagues found that a lack of regular care and comorbid illness among blacks was associated with a decision to defer surgery. ${ }^{20}$

Several observations in our analysis are worth noting. While metastatic disease was excluded from our analysis, we included T4 disease (i.e., that which has extended beyond the pancreas into nearby vasculature and nerves) which is widely considered unresectable. Although this accounts for nearly $20 \%$ of our study population and potentially skews the cohort of patients who were "recommended surgery" as being appropriate, it is worth noting that surgery was recommended in $20 \%$ of these patients suggesting that surgeons may be offering surgery where it is potentially not appropriate and not offering it when it is appropriate (in our sample, surgery was not recommended in $58 \%$ with $\mathrm{T} 0 / 1 / 2$ disease). Without comorbidity data and information about actual performance status in SEER, it is entirely possible that surgeons are in fact making appropriate recommendations to $\mathrm{T} 4$ and $\mathrm{T} 1 / 2$ patients, respectively. Relatedly, in order to account for the possibility that the large proportion of patients with T4 or TX disease mainly influenced the result of our study, we performed a sensitivity analysis excluding these patients: there were no significant changes to our conclusions.

Although we examined a large, representative cancer registry spanning 22 years, several limitations of our study are acknowledged. Despite including nearly 36,000 patients in our study, only 104 blacks and 588 whites refused surgery in our study. Race is often a proxy for other explanatory demographic variables, such as education, socioeconomic status, and marital status. ${ }^{21}$ Although our analyses are adjusted for rural or urban residence, education level, and poverty level, these data in SEER are based on ZIP code and census tract data; prior studies have demonstrated the validity of census tract measures as an approximation for individual socioeconomic status (SES). ${ }^{22}$ Information on medical comorbidities, which is not available in SEER, potentially influences a physician's decision to recommend surgery as well as a patient's decision whether or not to accept recommended treatment. ${ }^{20}$ Data regarding individual hospitals and providers were not available, precluding our ability to examine provider-specific trends. One explanation for the observed racial disparities in the present study is that black patients potentially cluster among physicians, such as primary care physicians, who may not refer to surgeons or have preconceived notions about the treatment of pancreatic cancer. Lastly, detailed information about the resectability of locally advanced pancreatic cancer is also not available in SEER. It is therefore impossible to further distinguish patients with locally advanced but still potentially resectable disease from patients with locally unresectable tumor burden. In light of the limitations presented, a national dataset consisting of a large cohort of patients undergoing pancreatic surgery is needed to identify subtle issues as discussed in this manuscript; more information about patient and provider characteristics would be desired.

Our analyses suggest that although racial disparities in the refusal of pancreatic cancer surgery have diminished over the past two decades, significant disparities in the recommendation and performance of surgery persist. Providers must strive to minimize potential racial biases in their treatment recommendations and attempt to better understand their patients' reasons for refusing surgery. It is unclear why blacks continue to be recommended surgical resection for pancreatic cancer less often than whites. While it is encouraging that the racial disparity in the refusal of pancreatic cancer surgery has decreased, it is likely that both provider- and patient-level factors have a substantial impact on surgery recommendation and its acceptance. The identification of such factors is critical to design a framework for eliminating disparities in cancer-directed surgery for pancreatic cancer. 


\section{References}

1. Siegel R, Naishadham D, Jemal A. Cancer statistics, 2012. CA: Cancer Journal for Clinicians. 62(1):10-29.

2. SEER. SEER Stat Fact Sheets: Pancreas [Internet]. Surveillance, Epidemiology, and End Results (SEER) Program. 2012 [cited 2012 Dec 10]. Available from: http://seer.cancer.gov/statfacts/html/pancreas.html

3. Fesinmeyer MD, Austin MA, Li CI, De Roos AJ, Bowen DJ. Differences in survival by histologic type of pancreatic cancer. Cancer Epidemiology, Biomarkers \& Prevention. 2005 Jul;14(7):176673.

4. Jinjuvadia R, Jinjuvadia K, Liangpunsakul S. Racial Disparities in Gastrointestinal Cancers-Related Mortality in the US Population. Digestive Diseases and Sciences. 2013 58:236-243, Jul 15

5. Murphy MM, Simons JP, Hill JS, McDade TP, Chau Ng S, Whalen GF, et al. Pancreatic resection: a key component to reducing racial disparities in pancreatic adenocarcinoma. Cancer. 2009 Sep 1;115(17):3979-90.

6. Eloubeidi MA, Desmond RA, Wilcox CM, Wilson RJ, Manchikalapati P, Fouad MM, et al. Prognostic factors for survival in pancreatic cancer: a population-based study. American Journal of Surgery. 2006 Sep;192(3):322-9.

7. SEER. SEER [Internet]. Surveillance, Epidemiology, and End Results (SEER) Program. 2012 [cited 2012 Dec 10]. Available from: http://seer.cancer.gov/

8. Amin S, McBride RB, Kline JK, Mitchel EB, Lucas AL, Neugut AI, et al. Incidence of subsequent pancreatic adenocarcinoma in patients with a history of nonpancreatic primary cancers. Cancer. 2012 Mar 1;118(5):1244-51.

9. National Cancer Institute. Center to Reduce Health Disparities. [Internet]. Available from: http://crchd.cancer.gov/

10. Agency for Healthcare Research and Quality. Activities to Reduce Racial and Ethnic Disparities in Health Care. [Internet]. Available from: http://www.ahrq.gov/qual/disparities.htm

11. Bilimoria KY, Ko CY, Tomlinson JS, Stewart AK, Talamonti MS, Hynes DL, et al. Wait times for cancer surgery in the United States: trends and predictors of delays. Annals of Surgery. 2011 Apr;253(4):779-85.
12. Moskowitz GB, Stone J, Childs A. Implicit stereotyping and medical decisions: unconscious stereotype activation in practitioners' thoughts about African Americans. American Journal of Public Health. 2012 May;102(5):996-1001.

13. Manfredi C, Kaiser K, Matthews AK, Johnson TP. Are racial differences in patient-physician cancer communication and information explained by background, predisposing, and enabling factors? Journal of Health Communication. 2010 Apr;15(3):272-92.

14. Hausmann LRM, Mor M, Hanusa BH, Zickmund S, Cohen PZ, Grant $\mathrm{R}$, et al. The effect of patient race on total joint replacement recommendations and utilization in the orthopedic setting. Journal of General Internal Medicine. 2010 Sep;25(9):982-8.

15. Bilimoria KY, Bentrem DJ, Ko CY, Stewart AK, Winchester DP, Talamonti MS. National failure to operate on early stage pancreatic cancer. Annals of Surgery. 2007 Aug;246(2):173-80.

16. McGhan LJ, Etzioni DA, Gray RJ, Pockaj BA, Coan KE, Wasif N. Underuse of curative surgery for early stage upper gastrointestinal cancers in the United States. Journal of Surgical Research. 2012 Sep;177(1):55-62.

17. Musa D, Schulz R, Harris R, Silverman M, Thomas SB. Trust in the health care system and the use of preventive health services by older black and white adults. American Journal of Public Health. 2009 Jul;99(7):1293-9.

18. Kennedy BR, Mathis CC, Woods AK. African Americans and their distrust of the health care system: healthcare for diverse populations. Journal of Cultural Diversity. 2007 Jan;14(2):56-60.

19. Cooper-Patrick L, Gallo JJ, Gonzales JJ, Vu HT, Powe NR, Nelson $\mathrm{C}$, et al. Race, gender, and partnership in the patient-physician relationship. JAMA. 1999 Aug 11;282(6):583-9.

20. Cykert S, Dilworth-Anderson P, Monroe MH, Walker P, McGuire FR, Corbie-Smith G, et al. Factors associated with decisions to undergo surgery among patients with newly diagnosed early-stage lung cancer. JAMA. 2010 Jun 16;303(23):2368-76.

21. Winker MA. Measuring race and ethnicity: why and how? JAMA. 2004 Oct 6;292(13):1612-4.

22. Schwartz KL, Crossley-May H, Vigneau FD, Brown K, Banerjee M. Race, socioeconomic status and stage at diagnosis for five common malignancies. Cancer Causes \& Control. 2003 Oct;14(8):761-6. 